\title{
Iliac crest free flap: Indications, tips and pitfalls
}

\author{
Paolo Cariati ${ }^{1}$, Susana Arroyo Rodriguez ${ }^{1}$, Daniel Pampin Ozan ${ }^{1}$, Andrea Ferri ${ }^{2}$, Silvano Ferrari ${ }^{2}$ \\ ${ }^{1}$ Department of Oral and Maxillofacial Surgery, Maxillofacial Surgeon, Hospital General Universitario Albacete, Albacete, Spain; ${ }^{2}$ Department of \\ Oral and Maxillofacial Surgery, Maxillofacial Surgeon, Ospedale Universitario Maggiore, Parma, Italy \\ Contributions: (I) Conception and design: All authors; (II) Administrative support: None; (III) Provision of study materials or patients: All authors; (IV) \\ Collection and assembly of data: P Cariati, S Ferrari; (V) Data analysis and interpretation: P Cariati; (VI) Manuscript writing: All authors; (VII) Final \\ approval of manuscript: All authors. \\ Correspondence to: Paolo Cariati. Hospital General Universitario Albacete, Calle Hermanos Falco, 37, 02006, Albacete, Spain. \\ Email: paolocariati1@gmail.com.
}

\begin{abstract}
Reconstruction of head and neck defects following oncological ablative surgery represents a considerable challenge for reconstructive surgeons. Common reconstruction options are the fibula, iliac crest, tip scapular and lateral scapula border free flap. The vascularized iliac crest free flap was first described by Taylor et al. in 1979 and it has been widely used for the reconstruction of composite head and neck defects. This flap guarantees excellent functional and aesthetic outcomes in the reconstruction of posterior segmental mandible defects. For the reconstruction of maxillary defects, it is important to note that the iliac crest bone shape usually allows for an adequate reconstruction without the need to conduct any osteotomies, and this substantially facilitates the flap inset. Thus, the iliac crest free flap offers a valuable reconstructive alternative that should be part of the armamentarium of all head and neck reconstructive surgeons. When this option is well indicated, the success rate, as well as functional and aesthetic outcomes, are comparable to other flaps used for reconstructing the maxillofacial area. The main aim of the present report is to analyze the importance of the DCIA flap for the reconstruction of complex defects of the maxillofacial region and to present possible applications, suggestions, and pitfalls associated with this technique.
\end{abstract}

Keywords: Deep circumflex iliac artery free flap; pedicle length; bone quality; head and neck reconstruction

Received: 10 July 2020; Accepted: 30 July 2020; Published: 14 August 2020.

doi: 10.21037/fomm-2020-mr-04

View this article at: http://dx.doi.org/10.21037/fomm-2020-mr-04

\section{Introduction}

Reconstruction of head and neck defects following oncological ablative surgery represents a considerable challenge for reconstructive surgeons. In this sense, composite bone and soft tissue defects need to be reconstructed with flaps that allow for restoring all the anatomical components that have been resected during extirpative surgery. These defects are usually reconstructed using an osteo-cutaneous free flap $(1,2)$. Versatility, reliability and effectiveness are key points for guiding the reconstructive surgeon when selecting the most appropriate flap for each case. Common reconstruction options are the fibula, iliac crest, tip scapular and lateral scapula border free flap. The vascularized iliac crest free flap was first described by Taylor et al. in 1979 and it has been widely used for the reconstruction of composite head and neck defects (3). However, recent years have seen a decline in popularity of this reconstructive resource, and it is rarely selected for head and neck reconstruction by the vast majority of reconstructive surgeons. The main reasons for the decrease in use of this flap include the relatively short pedicle length $(8-10 \mathrm{~cm})$, increased harvest time, and donor site morbidity (4). However, the microvascularized iliac crest flap has unique qualities that cannot be found in any other type of flap. First, deep circumflex iliac artery (DCIA) vessels are rarely affected by atherosclerosis and are usually of sufficient caliber for carrying microvascular anastomosis 

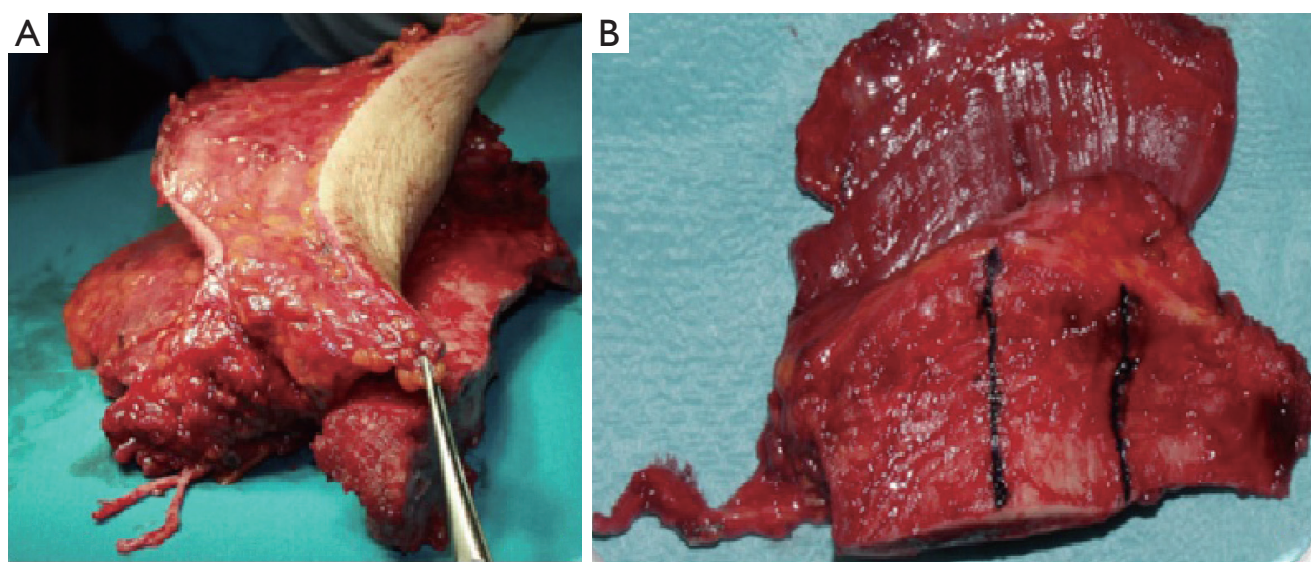

Figure 1 Composite osteo-cutaneous free DCIA flap. (A) With skin paddle. (B) without skin paddle.

in the cervical region. Second, the DCIA flap offers a large quantity of high-quality bone. Third, the DCIA flap ensures easy dental rehabilitation with osseointegrated implants (5). The main aim of the present report is to analyze the importance of the DCIA flap for the reconstruction of complex defects of the maxillofacial region and to present possible applications, suggestions, and pitfalls associated with this technique.

\section{Anatomy}

Bone and skin paddle are based in the DCIA and deep circumflex iliac vein (DCIV). These vessels arise from the external iliac vessels (5). The vascular pedicle of the internal oblique muscle is represented by the ascending branch of the deep circumflex iliac vessels. This branch is usually located approximately $2 \mathrm{~cm}$ medial to the anterior superior iliac spine (ASIS) and runs along the medial surface of the internal oblique muscle. The ascending branch arises in a plane between the transversus abdominis and the internal oblique muscle. The musculocutaneous perforators emerge through the transversus abdominis, internal oblique, and external oblique muscles along the iliac crest. The greatest concentration of these vessels can be found approximately 6 to $8 \mathrm{~cm}$ behind the ASIS. The skin paddle is obliquely oriented, with its major axis running along the iliac crest. The blood supply to the iliac crest bone is derived from the deep circumflex iliac vessels coursing along the inner aspect of the iliac crest in a groove between the iliacus and transversus abdominis muscles. This is located approximately $2 \mathrm{~cm}$ from the top of the crest (6).

\section{Surgical technique}

In this section we will describe the surgical technique used to harvest the iliac crest free flap without skin paddle, since this is the technique most frequently used in head and neck reconstruction (Figure 1). In fact, other types of flaps as fibula free flap, tip scapular free flap or lateral scapula border free flap should be considered in cases of composite defects with extensive skin loss.

The patient is put into the supine position and a small role is placed under the distal lumbar region. After identifying the femoral pulse, the first step is to make a vertical incision (approximately $5 \mathrm{~cm}$ ) over the femoral vessels extended approximately $2 \mathrm{~cm}$ above the inguinal ligament. The incision is then curved laterally with an oblique course to the ASIS. The completed incision simulates a reverse $\mathrm{L}$ shape with the long limb parallel to the inguinal ligament. The incision is continued through the skin and subcutaneous tissue to expose the external oblique muscle and its aponeurosis. The skin should be separated from the external oblique muscle until approximately 2 to $2.5 \mathrm{~cm}$ from the medial aspect of the crest. At this point, it is possible to identify dominant musculocutaneous perforators between the subcutaneous tissue and the external oblique muscle. These perforator vessels must be preserved when a skin paddle is required for the reconstruction (7).

The next step is to make an incision through the full thickness of the external oblique muscle, which is usually approximately $2.5 \mathrm{~cm}$ in width. It is important to note that the external oblique muscle should not be separated from the internal oblique muscle in a caudal direction in order to avoid damage to the perforator vessels in cases 
where surgeons are aiming to harvest an osteo-cutaneous flap. The next step is to expose the entire internal oblique muscle by elevating the external oblique muscle. Once the internal oblique muscle is completely exposed, it is then separated from the transversus abdominis muscle. The plane of dissection can easily be identified caudally to the twelfth rib. In fact, in this region a change in the direction of the muscle fibers allows the surgeon to identify the plane of dissection. The internal oblique muscle should be completely elevated from cephalad to caudad. During the elevation of the internal oblique muscle it is essential to maintain the integrity of the transversus abdominis muscle and its aponeurosis. Moreover, extreme care must be taken to preserve the ascending branch of the DCIA, which ensures the blood supply of the internal oblique muscle. The perforator vessels, originating from intercostal vessels, are usually identified between the transversus abdominis and internal oblique muscle. These vessels can be preserved in the cephalic area. However, the terminal branches of the intercostal vessels should be transected in the caudal region. Moreover, interconnecting branches between the ascending branch and the terminal branches of the intercostal vessels are often observed at this stage. It is extremely important to carefully coagulate these interconnecting branches with bipolar cautery. Unipolar cautery should be avoided in this phase. Usually, the DCIA and DICV are readily located by following the dissection of the ascending branch inferiorly and medially. The ascending branch usually joins the DCIA in the region medial to the ASIS (8).

After visualizing the union between the ascending branch and the DCIA, the next step consists of following the DCIA and DCIV until the point at which they are joined to their external iliac artery and vein. The lateral portion of the transversus abdominus muscle is then transected, leaving a muscular cuff of $2 \mathrm{~cm}$ attached to the iliac crest. This maneuver allows for exposing the iliacus muscle and the lateral femoral cutaneous nerve. The DCIA and DCIV run in a fibrous tunnel created by convergence of the fasciae of the transversus abdominus muscle and the iliacus. At this point, the iliacus muscle needs to be transected to expose the inner table of the ilium. A 2-cm cuff of iliacus should be left attached to the inner table to protect the DCIA and DCIV. Sharp dissection along the entire lateral border of the iliac crest provides exposure to the outer table of the ilium to perform the osteotomies. The DCIA and DCIV need to be dissected from the surrounding soft tissues in the region between their emergence from the external iliac artery and vein to the ASIS. At this stage, meticulous dissection is essential in order to preserve the lateral femoral cutaneous nerve. A 2-3 cm cuff of iliacus muscle is left attached to the ilium to protect the DCIA. The transection of the iliopsoas and sartorius muscles along the medial aspect of the ilium completes the soft tissue dissection. The gluteus medius, sartorius, and tensor fasciae latae are removed to expose the external cortex of the iliac crest. The next step is to perform the osteotomies. The osteotomies are conducted from the lateral side and deep retractors should be used to protect the abdominal contents when performing medial bone cuts. After the osteotomies have been completed and the bone is completely mobilized, the DCIA and DCIA are separated from the external iliac vessels.

The closure of the donor site is an extremely delicate step and should be meticulously performed to avoid ventral hernias of the abdominal content. The first step is to approximate the transversus abdominis muscle to the iliacus muscle. Drill holes could be required along the remaining iliac bone to ensure the hermetic closure of the transversus abdominus and the iliacus muscle. The use of a synthetic mash on the internal oblique defect might help to avoid ventral hernia during the immediate postoperative period (9).

\section{Pitfalls}

* The presence of previous surgeries or weakness of the abdominal wall might predispose the patient to the development of hernias. Another donor site should be selected in presence of these risk factors.

* The presence of previous surgery such as appendicectomy could compromise the ascending branch of the DCIA and, thus, the blood supply of internal oblique muscle. In these cases, the surgeon should choose either the contralateral side or another reconstructive alternative.

* Herniation of the abdominal contents could be more frequent when the transverse abdominal muscle is very thin. A synthetic mesh should be used to ensure adequate closure of the abdominal wall in these cases.

* Flap harvest could be particularly difficult in patients with a large abdominal circumference. An alternative reconstructive option should be considered in these cases.

* This flap should be avoided women of childbearing age. In fact, the use of this flap could weaken the abdominal wall and cause problems during delivery.

* Vital structures such as the femoral nerve or intraperitoneal contents might be damaged during flap harvest or 
closure.

* The skin paddle of iliac crest flap might fail for several reasons such as inadequate flap design (incorporation of an insufficient number of perforator vessels), tension or kinking of the perforator vessels (caused by the alteration of the relationship between bone and skin paddle) and insufficient muscular cuff (10).

\section{Advantages}

The main advantages of iliac crest free flap are:

* Height of the bone;

* Similar shape of the native maxilla and posterior mandible;

* Possibility for harvesting composite flaps (Internal Oblique muscle);

* Vascular anomalies are extremely rare;

* Possibility of working with two teams;

* Good quality of bone for performing dental rehabilitation with osseointegrated implants (11).

\section{Disadvantages}

* Donor site morbidity (chronic pain, ventral hernias, persistent lateral femoral paresthesia, sexual impotence);

* Short pedicle $(8-10 \mathrm{~cm})(12)$

* Small caliber vessels.

\section{Mandibular reconstruction}

With the iliac crest free flap, it is possible to harvest bone of up to $16 \mathrm{~cm}$ in length. This quantity of bone is usually sufficient to restore most ablative mandibular defects. However, partial or total mandibular reconstruction usually require the use of a fibula flap. Although iliac crest flap ensures a high-quality bone, multiple remodeling osteotomies are generally needed to shape the new mandible. Due to its length, width and height, iliac crest represents the optimal bone for carrying out dental rehabilitation with osseointegrated implants (Figures 2 and 3). Moreover, iliac crest bone has a rich blood supply due to the high quantity of cancellus bone. This could be extremely useful for the management of specific pathologies such as ORN.

\section{Indications}

* Segmental defects;
* Trophic and dentate mandible;
* Limited soft tissue defects;
* Good prognosis;
* Dental implant placement;
* Fibula not available.

\section{Maxillary reconstruction}

The shape of Iliac crest bone is very similar to the native maxilla due to its natural curvature. This feature makes this flap particularly useful for maxillary reconstruction. In fact, osteotomies are not usually required to remodel the iliac crest bone and adapt it to the recipient site. It is important to note that iliac crest bone should be inset vertically to ensure the adequate reconstruction of ablative defects of the upper jaw. In this regard, this flap offers sufficient bone to reconstruct extensive maxillary defects (Figure 4). Moreover, the internal oblique muscle is extremely useful for the reconstruction of palatal defects (13). Muscle metaplasia usually occurs very rapidly and internal oblique muscle generally acquires a texture and color very similar to the mucosa of the hard palate. Moreover, for maxillary reconstruction, the length, width and height of iliac crest bone facilitate dental rehabilitation using osseointegrated implants. The main limitation of iliac crest free flap for the reconstruction of maxillary defects is related the length of the pedicle. In fact, DCIA pedicle is rarely longer than 8-10 $\mathrm{cm}$ and therefore vein or arterial grafts might be necessary in some cases.

\section{Indications}

* High maxillary defect (Class II-III-IV of Brown's classification);

- Bilateral maxillectomy;

* No need for neck dissection;

* Limited soft tissue defects.

\section{Discussion}

DCIA free flap has been widely used for head and neck reconstruction for several years. However, this reconstructive alternative is losing popularity and it is now often considered as a second choice for the reconstruction of oncological defects of the maxillofacial area. Arguments against the use of this flap include short pedicle length, difficulties of harvesting, and donor site morbidity. Despite these considerations, several authors have pointed 

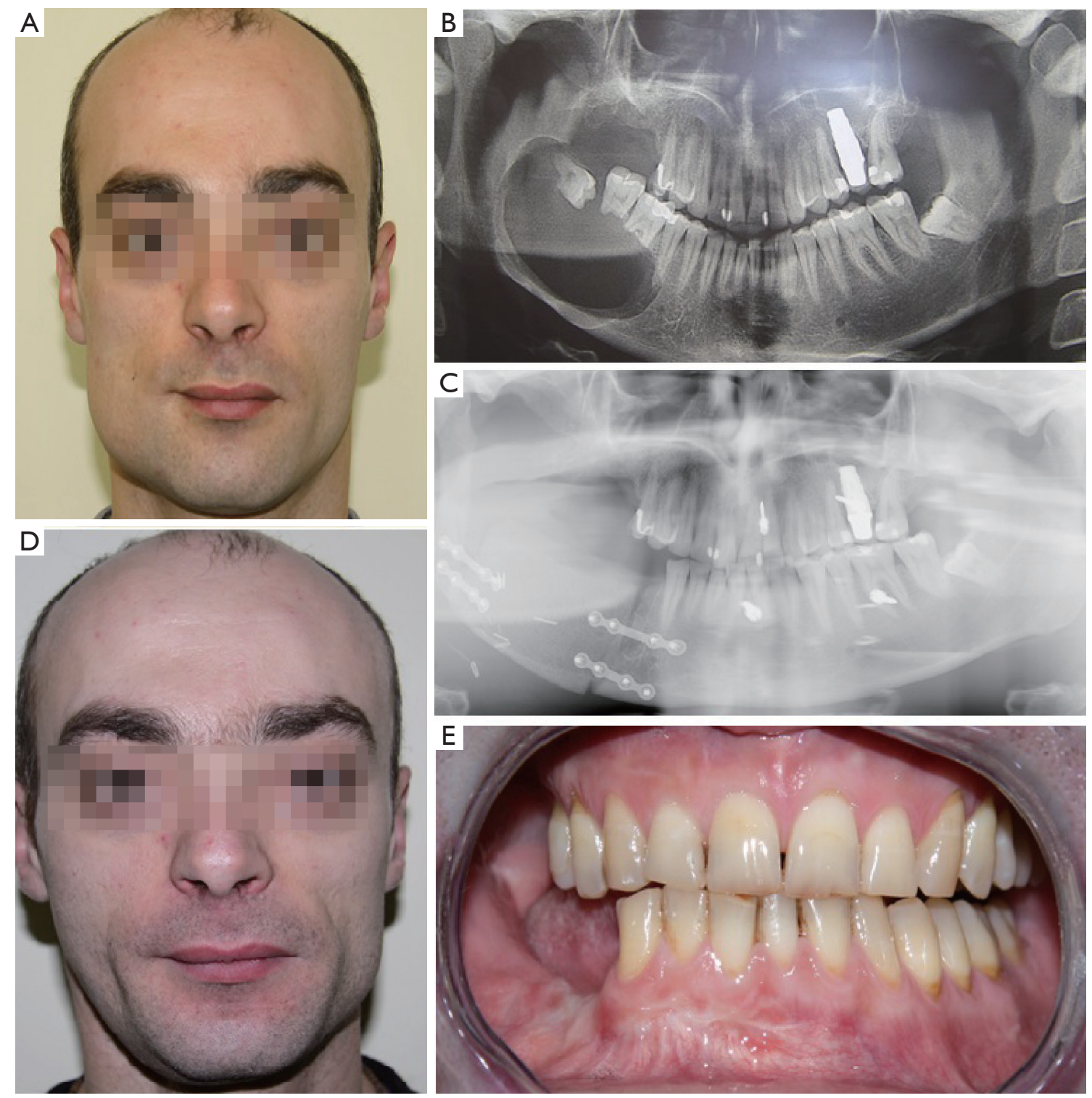

Figure 2 Patient 1. Mandibular reconstruction with DCIA free flap in a 28 years old male with ameloblastoma involving mandibular body and angle. (A) Patient before surgery. (B) OPG before ablative surgery. (C) Postoperative OPG. (D) Intraoral appearance after reconstruction. (E) Patient after reconstruction with DCIA free flap.

out the reliability and effectiveness of the vascularized iliac crest free flap. For instance, Zheng et al. reported a success rate of $95.6 \%$ with the use of DCIA free flap for oromandibular reconstruction (14). Similarly, Brown et al. reported the effectiveness of DCIA free flap for maxillary reconstruction (15). In our institution, DCIA free flap is routinely used for mandibular and maxillary reconstruction, and pedicle length or donor site morbidity have rarely been a problem. Specifically, we have carried out more than 60 reconstructions with DCIA free flap in the last 10 years. In our opinion, the indications are key when deciding to use this flap, and patients should be carefully selected. In fact, flap harvesting is extremely difficult in patients with a large abdominal circumference. Moreover, ventral hernias are also more frequent in this group of patients. Use of this flap should also be avoided in patients who have previously undergone surgery involving the abdominal wall. Another important aspect to bear in mind is the patient's oncological 

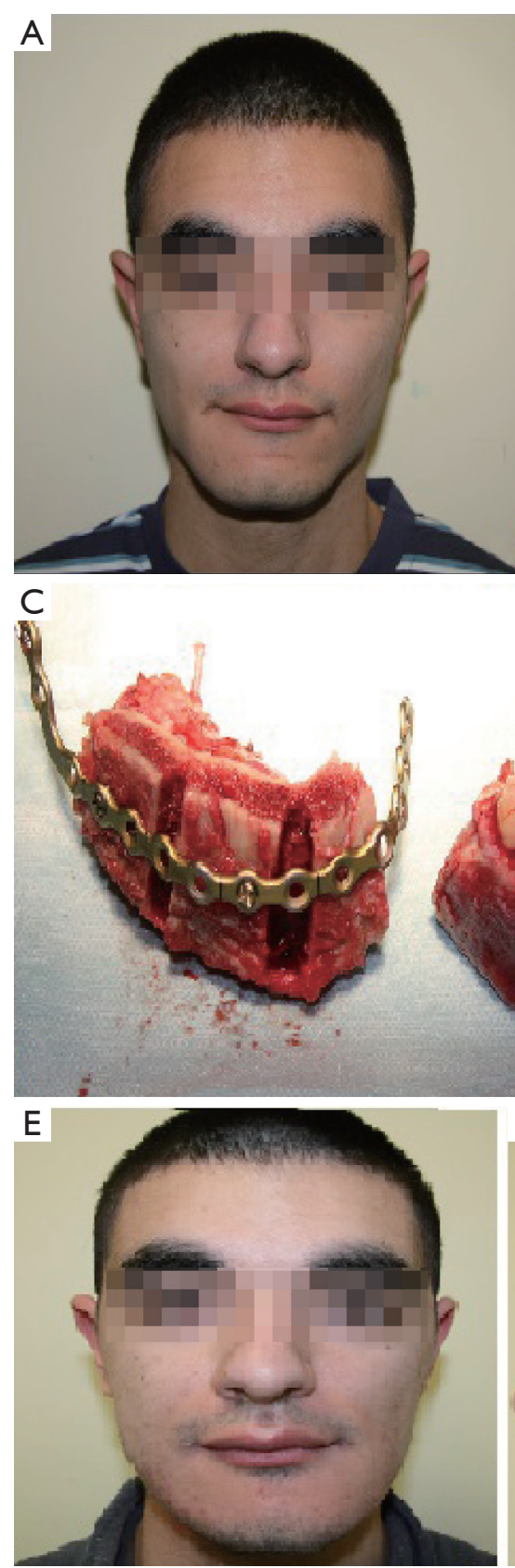
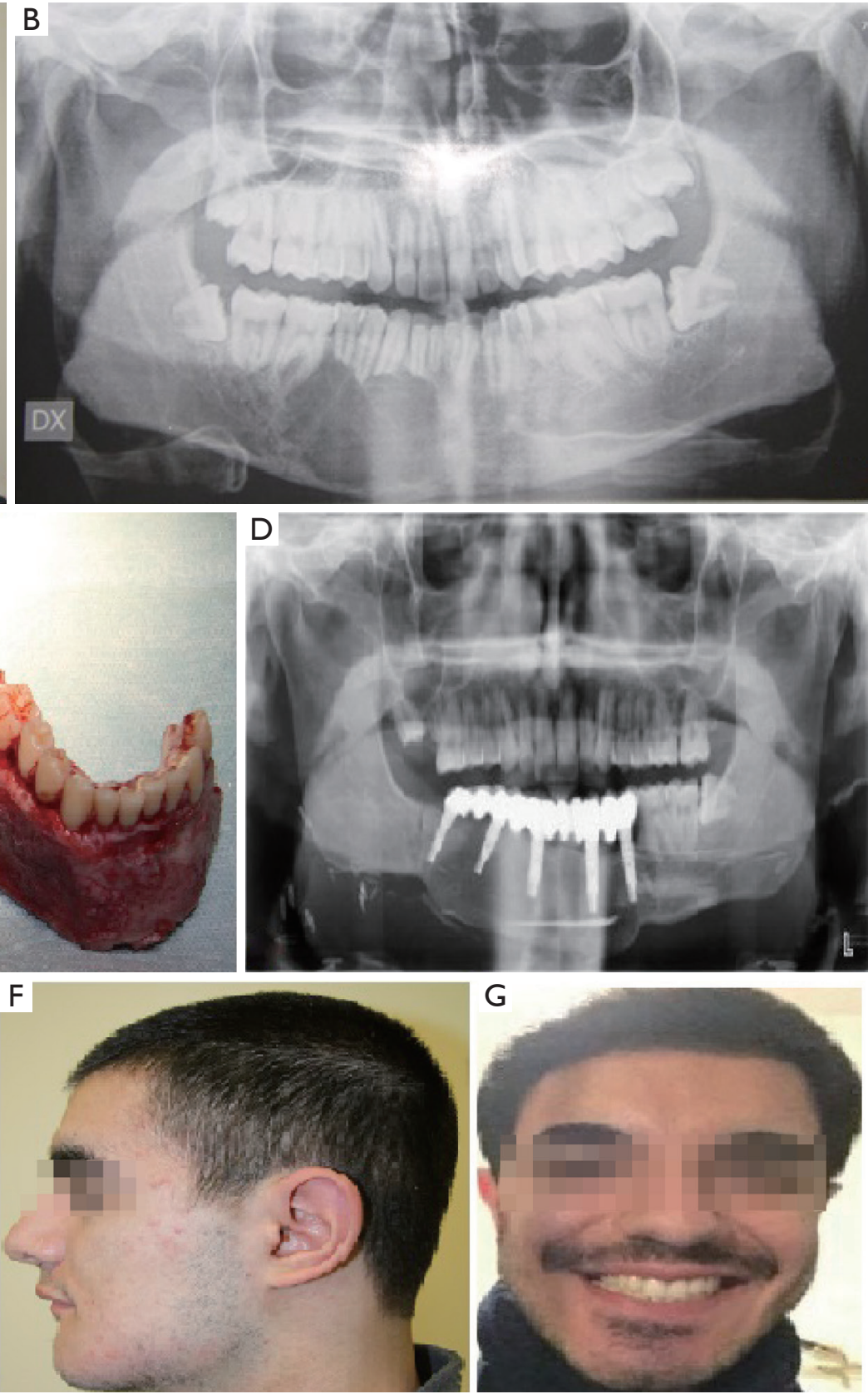

Figure 3 Patient 2. Mandibular reconstruction with DCIA free flap in a 16 years old male with mandibular ossifying fibromas. (A) Patient before surgery. (B) OPG before ablative surgery. (C) Clinical image showing the osteotomies of the iliac crest bone to resemble the native mandible. (D) OPG after dental implant placement. (E) Frontal view of patient after reconstructive surgery. (F) Profile view of patient after surgery. (G) Final patient clinical appearance after finish dental rehabilitation with osseointegrated implants.

history. In particular, pedicle length could be insufficient in a patient who has undergone previous neck dissection, and in these cases other free flaps such us the fibula flap or tip scapular flap should be considered. In this regard, we prefer to use the DCIA free flap for the reconstruction of benignant tumors involving the maxillofacial area. In the case of patients with a malignant tumor, the surgeon should be extremely meticulous during neck dissection, taking 

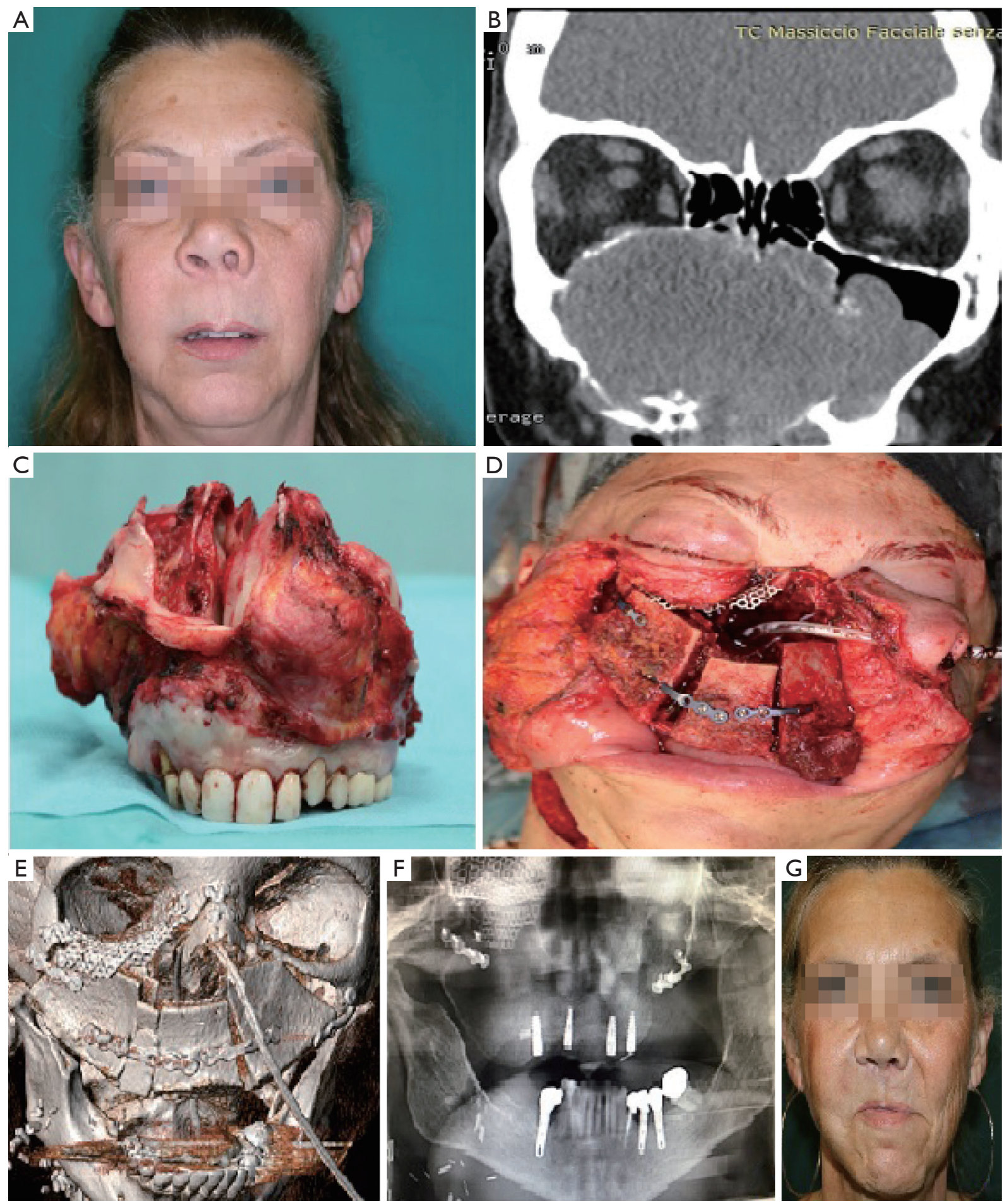

Figure 4 Patient 3. Maxillary reconstruction with DCIA free flap in a 50 years old female with a low grade leiomyosarcoma. (A) Patient before surgery. (B) Ct scan showing the extension of the tumor. (C) Tumorectomy. (D) Clinical image showing the osteotomies of the iliac crest bone to resemble the native maxilla. (E) Postoperative Ct scan. (F) Postoperative OPG after dental implant insertion. (G) Patient after reconstruction with DCIA free flap. 
particular care to preserve the facial vessels. Moreover, this flap could be more problematic in elderly patients for several reasons. First, this group of patients usually present a certain laxity and weakness of the abdominal muscles and this could predispose them to ventral hernias. Second, the need for immobilization in the immediate postoperative period (10-14 days) and ambulation problems related to flap harvest could represent a major problem for this group of patients. Third, the flow of the facial vessels could be insufficient in elderly patients. For these reasons, we prefer to use the DCIA free flap in young patients. At our institution, tip scapular flap or fibula flap are generally used for oncological reconstruction in patients aged $>65$ years.

Despite these drawbacks, the DCIA free flap presents unique qualities that make it particularly suitable for the reconstruction of composite defects of the maxillofacial area. For instance, no other bony flaps offer a comparable bone width and height. In this regard, the DCIA flap offers a bone width that could range from 1.5 to $3 \mathrm{~cm}$. Moreover, it is very important to stress that the surgeon can decide the bone height of the iliac crest flap according to the defect (bone height could range from 1.9 to $5 \mathrm{~cm}$ ). This feature is particularly important for maxillary reconstruction, and no other bony flaps allow for modifying the height of the bone components according to the patient's defect. For instance, the average bone eight of the fibula flap is approximately $1.5 \mathrm{~cm}$, which is substantially lower than the rest of the jaw, and this could cause numerous problems when conducting a dental rehabilitation with osseointegrated implants. However, the DCIA free flap represents the optimal subtract for the insertion of dental implants and generally ensures high-quality dental rehabilitation due to the considerable width and height of the iliac crest bone. Other important qualities of the microvascularized iliac crest flap are related to its shape. In particular, the shape of the iliac crest is very similar to the mandible angle and the native maxilla $(16,17)$. Because of these features, this flap guarantees excellent functional and aesthetic outcomes in the reconstruction of posterior segmental mandible defects. For the reconstruction of maxillary defects, it is important to note that the iliac crest bone shape usually allows for an adequate reconstruction without the need to conduct any osteotomies, and this substantially facilitates the flap inset.

Thus, the iliac crest free flap offers a valuable reconstructive alternative that should be part of the armamentarium of all head and neck reconstructive surgeons. When this option is well indicated, the success rate, as well as functional and aesthetic outcomes, are comparable to other flaps used for reconstructing the maxillofacial area.

\section{Acknowledgments}

Funding: None.

\section{Footnote}

Provenance and Peer Review: This article was commissioned by the Guest Editor (Paolo Cariati) for the series "Microvascular reconstruction of head and neck oncological defects-state of the art" published in Frontiers of Oral and Maxillofacial Medicine. The article has undergone external peer review.

Conflicts of Interest: All authors have completed the ICMJE uniform disclosure form (available at: https://fomm. amegroups.com/article/view/10.21037/fomm-2020$\mathrm{mr}-04 /$ coif). The series "Microvascular reconstruction of head and neck oncological defects—state of the art" was commissioned by the editorial office without any funding or sponsorship. Paolo Cariati served as the unpaid Guest Editor of the series and serves as an unpaid editorial board member of Frontiers of Oral and Maxillofacial Medicine from Apr 2020 to Mar 2022. The authors have no other conflicts of interest to declare.

Ethical Statement: The authors are accountable for all aspects of the work in ensuring that questions related to the accuracy or integrity of any part of the work are appropriately investigated and resolved.

Open Access Statement: This is an Open Access article distributed in accordance with the Creative Commons Attribution-NonCommercial-NoDerivs 4.0 International License (CC BY-NC-ND 4.0), which permits the noncommercial replication and distribution of the article with the strict proviso that no changes or edits are made and the original work is properly cited (including links to both the formal publication through the relevant DOI and the license). See: https://creativecommons.org/licenses/by-nc-nd/4.0/.

\section{References}

1. Brown JS, Lowe D, Kanatas A, et al. Mandibular reconstruction with vascularised bone flaps: a systematic review over 25 years. Br J Oral Maxillofac Surg 
2017;55:113-26.

2. Kakarala K, Shnayder Y, Tsue TT, et al. Mandibular reconstruction. Oral Oncol 2018;77:111-7.

3. Taylor GI, Townsend P, Corlett R. Superiority of the deep circumflex iliac vessels as the supply for free groin flaps. Plast Reconstr Surg 1979;64:595-604.

4. Urken ML. Composite free flaps in oromandibular reconstruction. Review of the literature. Arch Otolaryngol Head Neck Surg 1991;117:724-32.

5. Baliarsing AS, Kumar VV, Malik NA, et al. Reconstruction of maxillectomy defects using deep circumflex iliac arterybased composite free flap. Oral Surg Oral Med Oral Pathol Oral Radiol Endod 2010;109:e8-e13.

6. Urken M, Cheney ML, Blackwell KE et al. Atlas of Regional and Free Flaps for Head and Neck Reconstruction: Flap Harvest and Insetting. Second edition. Lippincott Williams \& Wilkins, 2012.

7. Kheradmand AA, Garajei A, Kiafar M, et al. Assessing the Anatomical Variability of Deep Circumflex Iliac Vessels in Harvesting of Iliac Crest-Free Flap for Mandibular Reconstruction. J Craniofac Surg 2016;27:e320-3.

8. Safak T, Klebuc MJ, Mavili E, et al. A new design of the iliac crest microsurgical free flap without including the "obligatory" muscle cuff. Plast Reconstr Surg 1997;100:1703-9.

9. Cobb R, Muscat K, Cascarini L, Fry A. Fibrin sealant (Tisseel $^{\mathrm{TM}}$ ) for mesh fixation in repair of the deep circumflex iliac artery (DCIA) free flap donor site. Br J Oral Maxillofac Surg 2017;55:748-9.

10. Urken ML, Vickery C, Weinberg H, et al. The internal oblique-iliac crest osseomyocutaneous free flap in oromandibular reconstruction. Report of 20 cases. Arch Otolaryngol Head Neck Surg 1989;115:339-49.

11. van Gemert JT, van Es RJ, Rosenberg AJ, et al. Free vascularized flaps for reconstruction of the mandible: complications, success, and dental rehabilitation. J Oral Maxillofac Surg 2012;70:1692-8.

12. Kheradmand AA, Garajei A, Kiafar M, et al. Assessing the Anatomical Variability of Deep Circumflex Iliac Vessels in Harvesting of Iliac Crest-Free Flap for Mandibular Reconstruction. J Craniofac Surg 2016;27:e320-3.

13. Bianchi B, Ferri A, Ferrari S, et al. Iliac crest free flap for maxillary reconstruction. J Oral Maxillofac Surg 2010;68:2706-13.

14. Zheng L, Lv X, Zhang J, et al. Deep circumflex iliac artery perforator flap with iliac crest for oromandibular reconstruction. J Craniomaxillofac Surg 2018;46;1263-7.

15. Brown JS, Jones DC, Summerwill A, et al. Vascularized Iliac Crest With Internal Oblique Muscle for Immediate Reconstruction After Maxillectomy Br J Oral Maxillofac Surg 2002;40:183-90.

16. Mertensa C, Deckera C, Engela M, et al. Early bone resorption of free microvascular reanastomized bone grafts for mandibular reconstruction A comparison of iliac crest and fibula grafts. J Craniomaxillofac Surg 2014;42:e217-23.

17. Liu Q, Shao Z, Shang Z. Mandibular Reconstruction using the deep circumflex iliac artery flap effect of the length of bone harvested on donor site morbidity. Br J Oral Maxillofac Surg 2019;57:778-81. doi: 10.21037/fomm-2020-mr-04

Cite this article as: Cariati P, Arroyo Rodriguez S, Pampin Ozan D, Ferri A, Ferrari S. Iliac crest free flap: Indications, tips and pitfalls. Front Oral Maxillofac Med 2020;2:15. 